\title{
Uso y competición por plantas alimenticias entre Pithecia aequatorialis (Primates: Pitheciidae) y otros animales en la Amazonía peruana
}

\section{Use and competition for food plants between Pithecia aequatorialis (Primates: Pitheciidae) and other animals in the Peruvian Amazonia}

\section{Elvis J. Charpentier ${ }^{1}$, Gabriel García ${ }^{1}$ y Rolando Aquino ${ }^{2}$}

\author{
1 Equipo Primatológico de Loreto, Iquitos, Perú. \\ 2 Facultad de Ciencias Biológicas, Universidad Nacional Mayor de San Marcos, Lima, Perú. \\ Email Elvis J. Charpentier: equiprimatesperu@yahoo.com.pe \\ Email Gabriel García: garcia.mendoza25@gmail.com \\ Email Rolando Aquino: raquinoy2005@yahoo.es
}

\begin{abstract}
Resumen
En este reporte proporcionamos información sobre plantas alimenticias del huapo negro (Pithecia aequatorialis) y sus competidores en bosque de altura de la microcuenca del río Itaya, Amazonía peruana. De mayo a noviembre del 2009 y de enero a abril del 2010 seguimos silenciosamente a dos grupos familiares con el propósito de registrar las plantas cuyos frutos forman parte de su dieta. Durante los contactos tuvimos 90 eventos alimenticios en 48 especies de plantas agrupadas en 24 familias. De ellas, 36 habitan en bosque de colina baja y el resto en bosque de terraza alta. La mayor diversidad de plantas alimenticias está agrupada en siete familias que en conjunto representaron el $60.4 \%$, sobresaliendo entre ellas las familias Moraceae (8 especies), Fabaceae ( 6 especies) y Annonaceae (4 especies). Los frutos en su mayoría fueron consumidos en estado maduro, siendo el mesocarpio el más apreciado (40\%). Entre los mamíferos, siete de los competidores fueron primates, siendo el principal el pichico pardo (Saguinus lagonotus) y entre las aves el tucán de cuello blanco (Ramphastos tucanus). Finalmente, los frutos de Pseudolmedia laevigata (Moraceae) fueron los más preferidos por los competidores (7 especies de mamíferos y 2 de aves).
\end{abstract}

Palabras claves: Primates; Pithecia aequatorialis; plantas alimenticias; partes consumidas; competidores.

\section{Abstract}

In this report, we provide information on food plants of the equatorial saki (Pithecia aequatorialis) and its competitors in highland forest of the micro-watershed of the Itaya River, Peruvian Amazonia. From May to November 2009 and from January to April 2010 we followed silently two family groups with the purpose to identify plants whose fruits are part of their diet. During the contacts we had 90 food events in 48 plant species grouped in 24 families. Of these, 36 live in low hill forest and the rest in high terrace forest. The highest diversity of food plants is grouped into seven families that together accounted for $60.4 \%$, among them Moraceae (8 species), Fabaceae ( 6 species) and Annonaceae (4 species) families. The fruits were mostly eaten in ripe state, being the mesocarp the most appreciated (40\%). Among mammals, seven of the competitors were primates, with the main competitor being the red-mantled saddle-back tamarin (Saguinus lagonotus) and among birds the White-throated toucan (Ramphastos tucanus). Finally, the fruits of Pseudolmedia laevigata (Moraceae) were the most preferred by competitors ( 7 species of mammals and 2 of birds).

Keywords: Primates; Pithecia aequatorialis; food plants; ingested parts; competitors.

Citación:

Charpentier E.J., G. García \& R. Aquino. 2015. Uso y competición por plantas alimenticias entre Pithecia aequatorialis (Primates: Pitheciidae) y otros animales en la Amazonía peruana. Revista peruana de biología 22(2): 225 - 232 (Octubre 2015). doi: http://dx.doi.org/10.15381/rpb. v22i 2.11356

Presentado: $\quad 11 / 02 / 2015$

Aceptado: $\quad 20 / 06 / 2015$

Publicado online: $14 / 10 / 2015$
Información sobre los autores:

EJCh, GG, RA: realizaron el diseño experimental, participaron en el trabajo de campo, analizaron los datos y redactaron el manuscrito.

Los autores no incurren en conflictos de intereses.

Fuentes de financiamiento: El presente estudio fue financiado por Amazon Center por the Environmental Education and Research (ACEER) 


\section{Introducción}

Entre los mamíferos, los primates forman parte de la comunidad de frugívoros, quienes participan en los procesos ecológicos como dispersores y control de semillas (depredación) y en la polinización, conllevando a restaurar y mantener el equilibrio de los bosques amazónicos (Kricher 2008, Estrada 2006).

En referencia a los primates que habitan en la Amazonía peruana, existen numerosos estudios, pero la mayoría trata sobre aspectos ecológicos, comportamiento, interacciones, uso de estratos, dinámica poblacional, entre otros; pero cuando se trata de recursos alimenticios, los estudios mayormente fueron orientados hacia Saguinus spp. (Garber 1993, Knogge et al. 2003), Callicebus lucifer (Kinzey 1976, 1977, Kinzey \& Gentry 1978) y Cacajao calvus ucayalii (Aquino \& Encarnación 1999, Bowler \& Bodmer 2011), para el resto de primates son muy escasos o simplemente no existen. En cuanto al género Pithecia, los escasos estudios sobre recursos alimenticios fueron para $P$. monachus (Heymann \& Bartecki 1990), P. Isabela (antes P. monachus) (Soini 1995), P. irrorata (Palminteri 2010, Palminteri et al. 2012) y $P$. aequatorialis (Aquino et al. 2013).

La limitada información en relación a este aspecto, en particular para $P$. aequatorialis nos motivó a la conducción de este estudio, cuyos objetivos estuvieron orientados a: identificar las plantas de cuyos frutos y otros órganos se alimentan este primate, determinar el periodo de fructificación e identificar a sus principales competidores. El estudio fue conducido desde mayo a noviembre del 2009 y de enero a abril del 2010 en la quebrada Yanayacu, afluente izquierdo del río Itaya; aquí proporcionamos los resultados obtenidos.

\section{Área de estudio}

El estudio se realizó en bosques de altura circundantes a la quebrada Yanayacu ( $4^{\circ} 13^{\prime} 04^{\prime \prime S}$ y $\left.73^{\circ} 42^{\prime} 32^{\prime \prime W}\right)$, afluente izquierdo del río Itaya (Fig. 1). En el área de estudio predominaron los bosques de terraza alta y de colina baja de moderado a fuertemente disectado con pendientes de hasta $40 \%$ de inclinación (Aquino et al. 2007).

La vegetación dominante de tipo varillal estuvo compuesto por árboles de fuste recto y entre $10 \mathrm{a} 25 \mathrm{~m}$ de altura, con algunos emergentes arriba de $30 \mathrm{~m}$ como pashaco (Parkia sp.), quinilla (Elaeoluma sp.), sachacaimito (Manilkara spp.) y machimango (Eschweilera spp.). Entre las palmeras sobresalieron por su relativa abundancia cashapona (Socratea exorrhiza), huacrapona (Iriartea deltoidea), ungurahui (Oenocarpus bataua) y chambira (Astrocaryum chambira). Sotobosque mayormente abierto y poblado algunas veces por palmeras de irapay (Lepidocaryum tenue) y en otras por plantas arbustivas y herbáceas perennes. Piso del bosque generalmente cubierto por un colchón de hojarascas, particularmente en terraza alta y cimas de la colina.

El bosque desde la orilla del río hasta $5.0 \mathrm{~km}$ monte adentro presentó serias alteraciones por la extracción de especies maderables y de hojas de L. tenue, así como por la existencia de numerosas trochas de cazadores, algunas recientemente abiertas, por lo que la fauna nos pareció escasa, mientras que más allá de los $5.0 \mathrm{~km}$ el bosque mostró un perfil menos alterado, pero igualmente fueron encontrados trochas y campamentos de cazadores en uso, aunque la presencia de animales silvestres fue relativamente mayor, entre ellos $P$. aequatorialis.

\section{Material y métodos}

Selección de los grupos familiares.- Como primer paso para el reconocimiento de las plantas alimenticias y luego la colecta del material biológico fue necesaria la selección de dos grupos familiares, con hábitat preferentemente en bosque de terraza alta y colina baja. Para este propósito se procedió con la búsqueda al interior del bosque mediante caminatas pausadas, tratando de localizarlos a través de sus vocalizaciones, ruidos característicos originados durante las actividades de locomoción y alimentación y ubicando árboles en fructificación.

La selección de los grupos se hizo tomando en cuenta los siguientes criterios: cercanía al campamento, facilidad para su localización y tamaño de grupo mayor a tres individuos, por lo que en este caso optamos por grupos de cinco y siete individuos, respectivamente.

Después de la selección de los grupos, con la ayuda de un GPS (Garmin 60) procedimos a la georreferenciación de los puntos de encuentro durante 15 días no consecutivos; los cuales nos permitieron conocer parcialmente el área domiciliar de cada grupo familiar. Basados en los puntos geo referenciados de cada encuentro, el siguiente paso fue la apertura de cinco transectos paralelos y cinco perpendiculares, todos de $1.5 \mathrm{~km}$ de longitud y distanciados en $100 \mathrm{~m}$, haciendo un total de 20 transectos; es decir, 10 en cada área domiciliar. Estos transectos sirvieron para la búsqueda y seguimiento de los grupos familiares por separado, para el cual contamos con la participación de dos equipos de observadores, cada uno conformado por un investigador y un guía de campo.

Debido a la alta presión de caza los grupos mostraron alta sensibilidad a la presencia del hombre que dificultó el seguimiento, de modo que el tiempo de contacto fue desde un mínimo de 5 minutos a un máximo de 30 minutos, factor que fue una limitante para la obtención de mayor información.

Colecta e identificación de plantas alimenticias.- Cada vez que hubo contacto se procedió al seguimiento sigiloso para

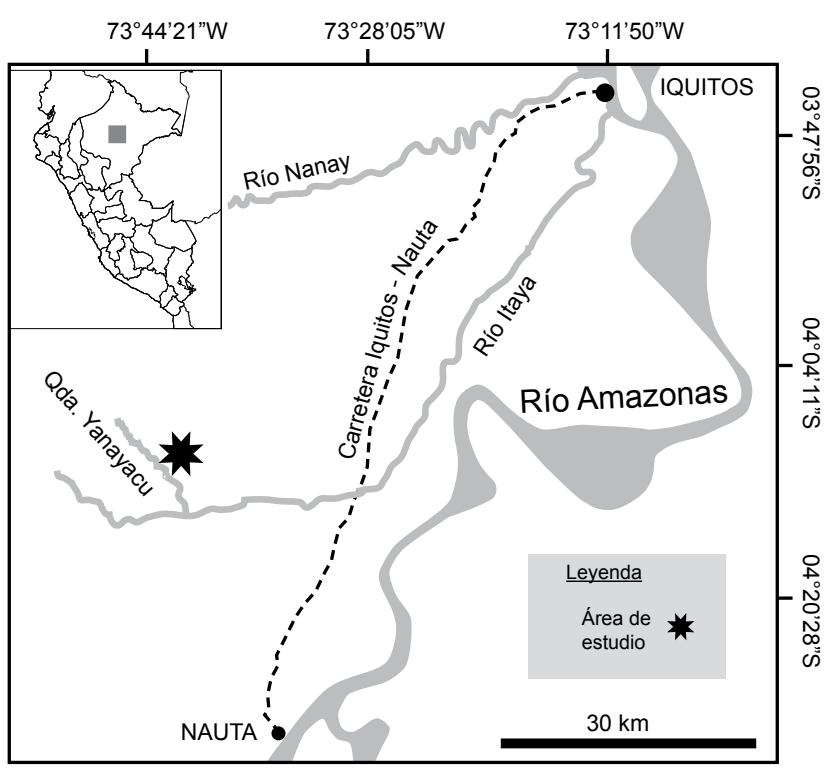

Figura 1. Mapa de la Microcuenca del río Itaya mostrando el área de estudio de Pithecia aequatorialis en la quebrada Yanayacu. 
la recolección de las partes de frutos y/o semillas que caían al piso durante la actividad alimenticia, para luego proceder a la ubicación del árbol afín de marcarlo con cinta plástica de color anaranjado, lo que facilitó la ubicación de la planta para la colecta del material biológico. Los restos de frutos y semillas caídos al piso fueron recolectados y llevados al campamento para la caracterización morfométrica, para luego colocarlos en frascos de plástico conteniendo alcohol al $70 \%$ para su preservación. Muestras botánicas de las plantas fueron colectadas siguiendo los procedimientos establecidos, luego puestos en papel periódico y finalmente en una bolsa plástica de $100 \times 50 \mathrm{~cm}$, donde fue añadido alcohol al $70 \%$.

Las muestras fueron colocadas en prensas portátiles para el traslado al Herbarium Amazonense de la Universidad Nacional de la Amazonia Peruana (UNAP) donde se procedió con el secado para el tratamiento taxonómico. La identificación se hizo por comparación con excicatas del Herbarium Amazonense y fueron registradas mediante el sistema de clasificación molecular del APG II (APG II 2003). Finalmente, las muestras identificadas fueron verificadas por un botánico especialista. Como complemento al registro e identificación de los frutos consumidos se anotó la fecha, partes consumidas (mesocarpio, arilo y semilla), estado del fruto (maduro e inmaduro), formas de vida de la planta (árbol, arbusto, liana, epífita) y frecuencia de consumo.

Adicionalmente registramos a los animales (mamíferos y aves) que compitieron por el recurso alimenticio con $P$. aequatorialis, incluyendo a los de hábito nocturno, para cuyo propósito fue necesario pernoctar cerca de las plantas alimenticias por más de dos noches lo que incluso nos permitió determinar la frecuencia de uso por estos competidores.

Análisis de datos.- Se elaboró una base de datos en Microsoft Excel 2010 de acuerdo a los objetivos del estudio; así, la lista de frutos consumidos por este primate y sus competidores fueron elaborados después de la identificación de las plantas, para luego utilizar la estadística descriptiva afín de plasmar los resultados en tablas y gráficos.

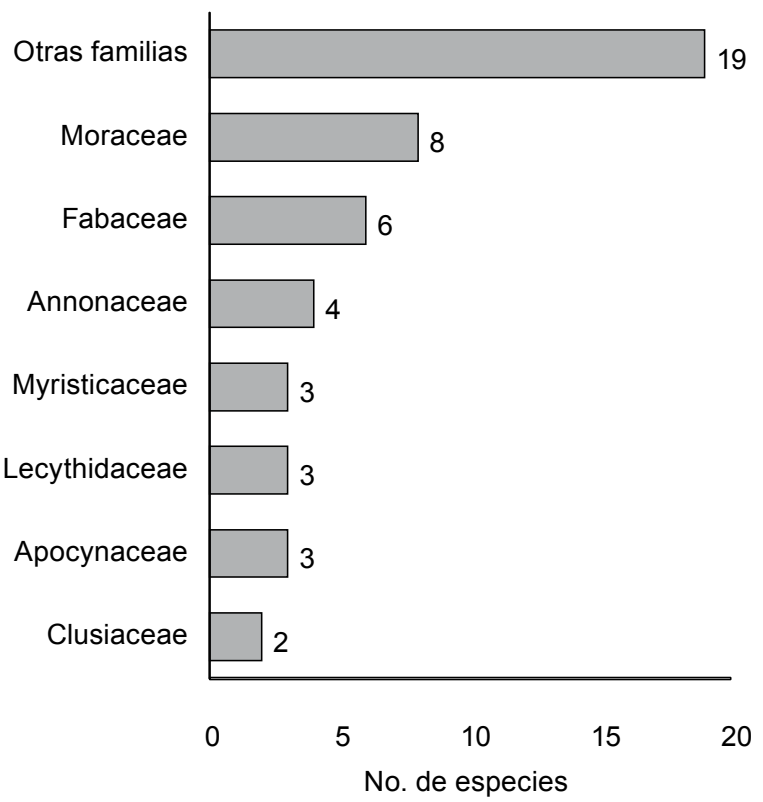

Figura 2. Especies de plantas alimenticias de Pithecia aequatorialis agrupadas por familias.

\section{Resultados}

Pithecia aequatorialis fue observado en 90 oportunidades alimentándose de los frutos de 48 especies de plantas agrupadas en 26 familias; de ellas, 32 fueron registradas en bosque de colina baja y solamente 12 en bosque de terraza alta, lo que indicaría que la mayor diversidad de plantas alimenticias para este primate en la Microcuenca del río Itaya se encuentra en el bosque de colina baja. Entre las especies, Parahancornia peruviana, Pseudolmedia laevigata, Iryanthera laevis y una especie no identificada de la familia Bromeliaceae fueron las únicas registradas en ambos tipos de bosques (Tabla 1).

La mayoría de las plantas se caracterizaron por ser de hábito arbóreo, por lo que los frutos generalmente se encontraban distribuidos por encima de los $15 \mathrm{~m}$ de altura. Por otro lado, la mayor diversidad de especies consumidas perteneció a siete familias que en conjunto sumaron el $60.4 \%$, sobresaliendo entre ellas Moraceae (8 especies), Fabaceae (6 especies) y Annonaceae (4 especies); el resto en su mayoría estuvo representado por una sola especie (Fig. 2). Entre las plantas alimenticias, Oenocarpus bataua (Arecaceae), Schefflera morototoni (Araliaceae), P. laevigata (Moraceae), Brosimun utile (Moraceae), Abuta grandifolia (Menispermaceae) y Drypetes sp. (Putranjivaceae) destacaron por su mayor frecuencia de consumo (Tabla 1).

Entre los frutos consumidos por este primate, el $79 \%$ fueron maduros y el $2 \%$ tanto maduro como inmaduro y correspondió únicamente a $O$. bataua (Fig. 3). Los resultados también indican que $P$. aequatorialis se alimentó principalmente del

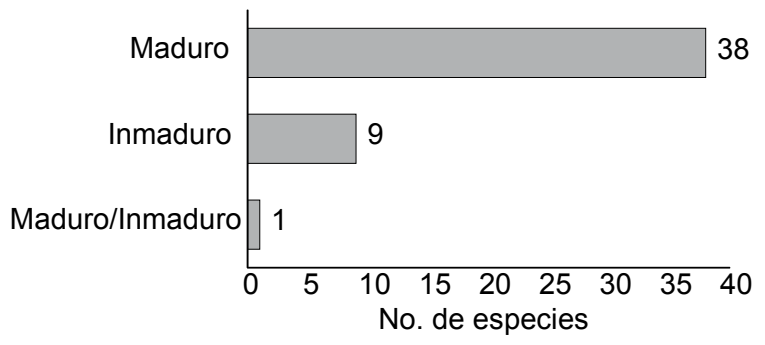

Figura 3. Estado de madurez de los frutos consumidos por Pithecia aequatorialis.

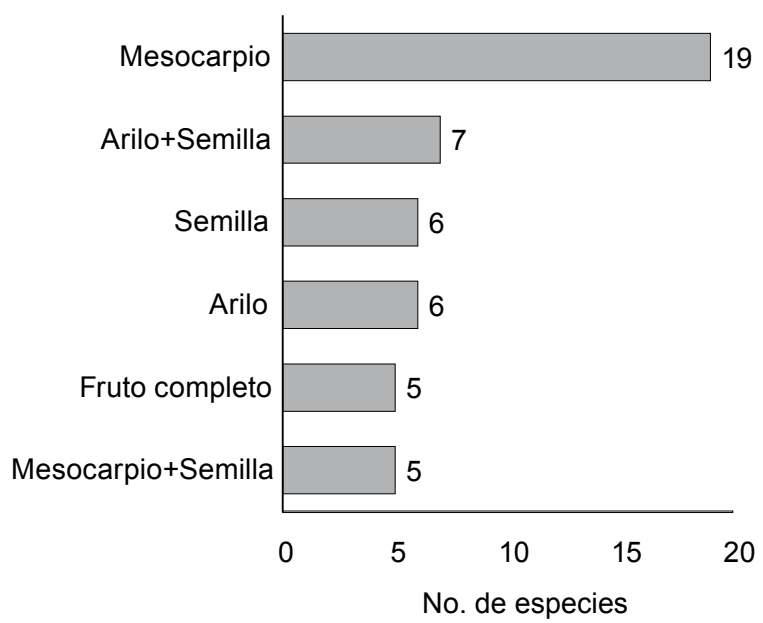

Figura 4. Proporción de las partes de frutos consumidos por Pithecia aequatorialis. 
Tabla 1. Plantas alimenticias de Pithecia aequatorialis registradas durante el período de estudio en bosques de colina baja y terraza alta. A: Árbol; Ar: Arbusto; L: Liana; P: Palmera; Ep.: Epífita.

\begin{tabular}{|c|c|c|c|c|c|}
\hline \multirow{2}{*}{ Familia } & \multirow{2}{*}{ Especies } & \multirow{2}{*}{ Formas de vida } & \multicolumn{2}{|c|}{ Tipo de bosque } & \multirow{2}{*}{$\begin{array}{l}\text { Frecuencia de } \\
\text { consumo }\end{array}$} \\
\hline & & & Terraza alta & Colina baja & \\
\hline \multirow{4}{*}{ Annonaceae } & Diclinanona calycina & $\mathrm{A}$ & $\mathrm{X}$ & & 1 \\
\hline & Duguetia spixiana & A & & $\mathrm{X}$ & 1 \\
\hline & Xylopia micans & A & $\mathrm{X}$ & & 2 \\
\hline & Xylopia sp. & $\mathrm{A}$ & & $\mathrm{X}$ & 3 \\
\hline \multirow{3}{*}{ Apocynaceae } & Couma macrocarpa & A & & $\mathrm{X}$ & 4 \\
\hline & Parahancornia peruviana & A & $\mathrm{X}$ & $\mathrm{X}$ & 3 \\
\hline & Rhigospira cuadrangularis & A & & $\mathrm{X}$ & 2 \\
\hline Anacardiaceae & Tapirira retusa & A & $\mathrm{X}$ & & 1 \\
\hline Arecaceae & Oenocarpus bataua & $\mathrm{P}$ & $\mathrm{X}$ & & 6 \\
\hline Araliaceae & Schefflera morototoni & A & & $\mathrm{X}$ & 5 \\
\hline Bombacaceae & Matisia sp. & $\mathrm{A}$ & & $\mathrm{X}$ & 2 \\
\hline Bromeliaceae & NI & Ep & $\mathrm{X}$ & $\mathrm{X}$ & 2 \\
\hline Burseraceae & Protium ferrugineum & $\mathrm{Ar}$ & & $\mathrm{X}$ & 1 \\
\hline \multirow{2}{*}{ Clusiaceae } & Clusia amazonica & $\mathrm{L}$ & $\mathrm{X}$ & & 1 \\
\hline & Garcinia macrophylla & A & & $\mathrm{X}$ & 1 \\
\hline Combretaceae & Buchenavia macrophylla & A & & $\mathrm{X}$ & 1 \\
\hline Euphorbiaceae & Senefeldera inclinata & $\mathrm{Ar}$ & & $\mathrm{X}$ & 1 \\
\hline \multirow{6}{*}{ Fabaceae } & Inga edulis & A & & $\mathrm{X}$ & 3 \\
\hline & Inga punctata & A & & $\mathrm{X}$ & 2 \\
\hline & Inga sp.1 & A & & $\mathrm{X}$ & 1 \\
\hline & Inga sp. 2 & A & & $\mathrm{X}$ & 1 \\
\hline & Inga sp. 3 & A & & $\mathrm{X}$ & 1 \\
\hline & Swartzia sp. & A & & $\mathrm{X}$ & 1 \\
\hline Humiriaceae & Sacoglottis sp. & A & $\mathrm{X}$ & & 3 \\
\hline Icacinaceae & Pleurisanthes sp. & $\mathrm{L}$ & & $\mathrm{X}$ & 1 \\
\hline Lacistemataceae & Lacistema aggregatum & A & & $\mathrm{X}$ & 1 \\
\hline \multirow{3}{*}{ Lecythidaceae } & Eschweilera parvifolia & A & & $\mathrm{X}$ & 1 \\
\hline & Eschweilera coriacea. & A & & $\mathrm{X}$ & 1 \\
\hline & Eschweilera sp. & A & & $\mathrm{X}$ & 1 \\
\hline Malvaceae & Theobroma subincanum & $\mathrm{Ar}$ & $\mathrm{X}$ & & 1 \\
\hline Marcgraviaceae & Marcgravia sp. & $\mathrm{L}$ & & $\mathrm{X}$ & 1 \\
\hline Menispermaceae & Abuta grandifolia & $\mathrm{L}$ & & $\mathrm{X}$ & 4 \\
\hline \multirow{8}{*}{ Moraceae } & Brosimum potabile & A & & $\mathrm{X}$ & 1 \\
\hline & Brosimun utile & A & & $\mathrm{X}$ & 4 \\
\hline & Brosimun rubescens & A & & $\mathrm{X}$ & 3 \\
\hline & Brosimun sp. 1 & A & & $\mathrm{X}$ & 1 \\
\hline & Brosimun sp. 2 & A & & $\mathrm{X}$ & 1 \\
\hline & Helicostylis tomentosa & A & & $\mathrm{X}$ & 1 \\
\hline & Naucleopsis mello-barretoi & A & $\mathrm{X}$ & & 1 \\
\hline & Pseudolmedia laevigata & $\mathrm{A}$ & $\mathrm{X}$ & $\mathrm{X}$ & 5 \\
\hline \multirow{3}{*}{ Myristicaceae } & Iryanthera laevis & A & $\mathrm{X}$ & $\mathrm{X}$ & 2 \\
\hline & Iryanthera tricornis & A & & $\mathrm{X}$ & 2 \\
\hline & Virola pavonis & A & & $\mathrm{X}$ & 1 \\
\hline Ochnaceae & Quiina klugii & $\mathrm{Ar}$ & $\mathrm{X}$ & & 1 \\
\hline Passifloracea & Dilkea retusa & $\mathrm{L}$ & $\mathrm{X}$ & & 1 \\
\hline Poligonaceae & Coccoloba sp. & $\mathrm{L}$ & $\mathrm{X}$ & & 1 \\
\hline Putranjivaceae & Drypetes sp. & A & & $\mathrm{X}$ & 4 \\
\hline Sapotaceae & Chrysophyllum prieuri & $\mathrm{A}$ & $\mathrm{X}$ & & 1 \\
\hline
\end{tabular}


Tabla 2. Partes de los frutos consumidos por Pithecia aequatorialis y período de consumo en el área de estudio. M: Mesocarpio; A: Arilo; S: Semilla, Fe: Fruto entero; 1: Maduro; 2: Inmaduro.

\begin{tabular}{|c|c|c|c|c|c|c|c|c|c|c|c|c|c|c|c|}
\hline \multirow{2}{*}{ Especies } & \multicolumn{4}{|c|}{ Parte consumida } & \multicolumn{11}{|c|}{ Período de consumo } \\
\hline & $\mathrm{M}$ & A & $S$ & $\mathrm{Fe}$ & $\mathrm{E}$ & $\mathrm{F}$ & $\mathrm{M}$ & A & $\mathrm{M}$ & $\mathrm{J}$ & $\mathrm{J}$ & A & $S$ & $\mathrm{O}$ & $\mathrm{N}$ \\
\hline Diclinanona calycina & $1 x$ & & & & $x$ & & & & & & & & & & \\
\hline Duguetia spixiana & $1 x$ & & & & & & & $x$ & & & & & & & \\
\hline Xylopia micans & & & $2 x$ & & & & & & & & & & $x$ & & \\
\hline Xylopia sp. & $1 x$ & & & & & & & & & & & & & $x$ & $x$ \\
\hline Couma macrocarpa & $1 x$ & & & & $x$ & & $x$ & & $x$ & $x$ & & & & & \\
\hline Parahancornia peruviana & $1 x$ & & & & & $x$ & & & $x$ & $x$ & $x$ & & & & \\
\hline Rhigospira cuadrangularis & $1 x$ & & & & & & & & & $x$ & $x$ & & & & \\
\hline Tapirira retusa & $2 x$ & & & & & & & & & & & & & & $x$ \\
\hline Oenocarpus bataua & $1 x / 2 x$ & & $1 x / 2 x$ & & & & & & & $x$ & $x$ & $x$ & $x$ & $x$ & \\
\hline Schefflera morototoni & $1 x$ & & $1 x$ & & & & & & & & & $x$ & $x$ & & \\
\hline Matisia sp. & & & $1 x$ & & & $x$ & & & & & & & & & \\
\hline Bromeliaceae & & & & $1 x$ & & & & & & & & & & & \\
\hline Protium ferrugineum & $1 x$ & & & & & & $x$ & & & & & & & & \\
\hline Clusia amazonica & & & & $1 x$ & & & & & & & $x$ & & & & \\
\hline Garcinia macrophylla & $1 x$ & & & & & & & $x$ & & & & & & & \\
\hline Buchenavia macrophylla & $2 x$ & & & & & & $x$ & & & & & & & & \\
\hline Senefeldera inclinata & & & $2 x$ & & $x$ & & & & & & & & & & \\
\hline Inga edulis & & $1 x$ & & & & $x$ & & & & & & & & & \\
\hline Inga punctata & & $1 x$ & & & $x$ & $x$ & & & & & & & & & \\
\hline Inga sp.1 & & $1 x$ & & & & & $x$ & & & & & & & & \\
\hline Inga sp.2 & & $1 x$ & & & & & & $x$ & & & & & & & \\
\hline Inga sp.3 & & $1 x$ & & & & & & $x$ & & & & & & & \\
\hline Swartzia sp. & & $1 x$ & $1 x$ & & & & & & & & & & $x$ & & \\
\hline Sacoglottis sp. & $1 x$ & & & & & & & & & & $x$ & & & & \\
\hline Pleurisanthes sp. & $1 x$ & & & & & & & & & & & & $x$ & & \\
\hline Lacistema aggregatum & & & & $1 x$ & & & & & & & & & $x$ & & \\
\hline Eschweilera parvifolia & & & $2 x$ & & $x$ & & & & & & & & & & \\
\hline Eschweilera coriacea & & & $2 x$ & & & & $x$ & & & & & & & & \\
\hline Eschweilera sp. & & & $2 x$ & & & & & $x$ & & & & & & & \\
\hline Theobroma subincanum & & $1 x$ & & & & & $x$ & & & & & & & & \\
\hline Marcgravia sp. & $1 x$ & & $1 x$ & & & & & $x$ & & & & & & & \\
\hline Abuta grandifolia & $1 x$ & & & & & & $x$ & & & & & & & & \\
\hline Brosimum potabile & $1 x$ & & $1 x$ & & & & & & & & & & $x$ & $x$ & \\
\hline Brosimun utile & $1 x$ & & $1 x$ & & & & & & & & & & & $x$ & $x$ \\
\hline Brosimun rubescens & $1 x$ & & $1 x$ & & & & & & & & & & & $x$ & \\
\hline Brosimun sp.1 & $2 x$ & & & & & & & & & & & & & $x$ & \\
\hline Brosimun sp.2 & $2 x$ & & $2 x$ & & & & & & & & & & & $x$ & \\
\hline Helicostylis tomentosa & $1 x$ & & & & & & & & $x$ & & & & & & \\
\hline Naucleopsis mello-barretoi & & $1 x$ & $1 x$ & & & & & & & & & & & & $x$ \\
\hline Pseudolmedia laevigata & & & & $1 x$ & & & & & & & & & & $x$ & \\
\hline Iryanthera laevis & & $1 x$ & $1 x$ & & & $x$ & & & & & & & & & \\
\hline Iryanthera tricornis & & $1 x$ & $1 x$ & & $x$ & $x$ & & & & & & & & & \\
\hline Virola pavonis & & $1 x$ & $1 x$ & & & & $x$ & & & & & & & & \\
\hline Quiina klugii & $1 x$ & & & & & & & & & & & $x$ & & & \\
\hline Dilkea retusa & $1 x$ & & & & $x$ & & & & & & & & & & \\
\hline Coccoloba sp. & & $1 x$ & $1 x$ & & & $x$ & & & & & & & & & \\
\hline Drypetes sp. & & & & $1 x$ & & & & & & & & & $x$ & $x$ & \\
\hline Chrysophyllum prieuri & & $1 x$ & $1 x$ & & & & & & & $x$ & & & & & \\
\hline
\end{tabular}


Tabla 3. Mamíferos y aves competidores por los recursos alimenticios de Pithecia aequatorialis en el área de estudio.

\begin{tabular}{|c|c|c|c|}
\hline Especies competidoras & $\begin{array}{l}\mathrm{N}^{\circ} \text {. de especies } \\
\text { compartidas }\end{array}$ & $\%$ & Plantas alimenticias \\
\hline \multicolumn{4}{|l|}{ Mamíferos: } \\
\hline Saguinus lagonotus & 11 & 23 & $\begin{array}{l}\text { Diclinanona calycina, Xilopia sp., Couma macrocarpa, Schefflera morototoni, } \\
\text { Inga edulis, Inga punctata, Eschweilera sp., Abuta grandifolia, Helicostylis } \\
\text { tomentosa, Coccoloba sp., Pseudolmedia laevigata }\end{array}$ \\
\hline Saimiri macrodon & 2 & 4 & Schefflera morototoni, Inga punctata \\
\hline Callicebus discolor & 5 & 10 & $\begin{array}{l}\text { Xilopia sp., Xilopia micans, Oenocarpus bataua, Inga punctata, Brosimum } \\
\text { potabile }\end{array}$ \\
\hline Callicebus lucifer & 6 & 12 & $\begin{array}{l}\text { Couma macrocarpa, Garcinia macrophylla, Drypetes sp., Brosimun utile, } \\
\text { Pseudolmedia laevigata, Virola pavonis }\end{array}$ \\
\hline Sapajus macrocephalus & 1 & 2 & Oenocarpus bataua \\
\hline Cebus yuracus & 2 & 4 & Oenocarpus bataua, Pseudolmedia laevigata \\
\hline Lagothrix poeppigii & 4 & 8 & $\begin{array}{l}\text { Couma macrocarpa, Oenocarpus bataua, Lacistema aggregatum, Pseudolmedia } \\
\text { laevigata }\end{array}$ \\
\hline Potos flavus & 5 & 10 & $\begin{array}{l}\text { Xilopia micans, Schefflera morototoni, Abuta grandifolia, Brosimum potabile, } \\
\text { Pseudolmedia laevigata }\end{array}$ \\
\hline Eira barbara & 1 & 2 & Pseudolmedia laevigata \\
\hline Sciurus sp. & 1 & 2 & Pseudolmedia laevigata \\
\hline Quirópteros & 1 & 2 & Marcgravia sp. \\
\hline \multicolumn{4}{|l|}{ Aves: } \\
\hline Ramphastos tucanus & 8 & 17 & $\begin{array}{l}\text { Parahancornia peruviana, Oenocarpus bataua, Schefflera morototoni, Abuta } \\
\text { grandifolia, Brosimun utile, Pseudolmedia laevigata, Iryanthera laevis, Virola } \\
\text { pavonis. }\end{array}$ \\
\hline Pteroglossus sp. & 1 & 2 & Oenocarpus bataua \\
\hline Penelope jacquacu & 1 & 2 & Schefflera morototoni \\
\hline Amazona sp. & 3 & 6 & Parahancornia peruviana, Inga edulis, Pseudolmedia laevigata \\
\hline Psarocolius sp. & 1 & 2 & Schefflera morototoni \\
\hline
\end{tabular}

mesocarpio maduro (40\%), en tanto que la semilla, arilo+semilla y mesocarpio+semilla fueron consumidos en proporciones similares que en conjunto sumaron el 33\% (Tabla 2, Fig. 4), excepto en Inga spp. y Theobroma subincanum que fueron consumidos únicamente el arilo, mientras que frutos enteros apenas representaron el 10\% y éstos correspondieron a Dripetes sp., Lacistema aggregatum (Lacistemataceae), Clusia amazónica (Clusiaceae), P. laevigata (Moraceae) y una bromeliaceae no identificada, los mismos que se caracterizaron por ser pequeńos, suaves y de sabor agradable.

La producción de frutos fue durante todo el año, pero la mayor diversidad de especies consumidas por este primate ocurrió entre enero-abril y setiembre-noviembre (Tabla 2). Los resultados también indican que el consumo de frutos de la gran mayoría de especies fue entre uno a dos meses, excepto $O$. bataua, Couma macrocarpa y $P$. peruviana que fueron consumidos durante 4 o 5 meses.

Pithecia aequatorialis compartió 22 especies de plantas alimenticias con 11 de mamíferos y 4 de aves (Tabla 3). Entre los mamíferos, los primates fueron los principales competidores, en particular S. lagonotus, quien se alimentó de los frutos de 11 especies equivalente al $23 \%$ de las plantas registradas para $P$. aequatorialis, mientras que entre las aves, Rhamphastus tucanus fue el otro competidor más importante al alimentarse de ocho especies equivalente al 17\%. Entre las especies de plantas, las más requeridas por los competidores fueron frutos de P. laevigata ( 7 especies de mamíferos y 2 de aves) y de $O$. bataua y $S$. morototoni (ambas con 6 especies entre mamíferos y aves), mientras que las menos apreciadas conforman la mayoría de las plantas, en particular Diclinanona calycina (Annonaceae), Garcinia macrophylla (Clusiaceae) y Drypetes sp.

En adición a las plantas alimenticias registradas, durante el seguimiento a uno de los grupos de $P$. aequatorialis se observó en dos oportunidades comiendo hojas tiernas de árboles no identificados y en otra ocasión un individuo adulto comiendo termitas aladas cuyo panal se encontraba en un árbol de tornillo (Cedrelinga catenaeformis).

\section{Discusión}

De acuerdo con la información bibliográfica disponible, las especies del género Pithecia no solo se alimentan de frutos, sino también de hojas, flores, exudados, insectos, miel, aves pequeñas y mamíferos pequeños como ratones y murciélagos (Izawa 1975, Oliveira et al. 1985, Soini 1995, Riveros \& Ferreira 2001, Aquino \& Bodmer 2004). No obstante, estos primates son considerados como eminentemente frugívoros, porque los frutos conforman un alto porcentaje en su régimen alimenticio (Buchanan et al. 1981, Soini 1995, Peres 1993, Norconk 2007, Aquino \& Bodmer 2004), coincidiendo así con los resultados obtenidos. En efecto, durante el período de estudio, los dos grupos de $P$. aequatorialis se alimentaron principalmente de frutos, en particular de especies pertenecientes a las familias Moraceae y Fabaceae, coincidiendo así con los resultados de 
Soini (1995) para P. isabela en bosque inundable de la Reserva Nacional Pacaya-Samiria, Peres (1993) para P. albicans en bosque de tierra firme de Tefé, Amazonas y Aquino \& Bodmer (2004) para P. irrorata en bosque de tierra firme del Sur oriente peruano. Otros estudios realizados en Pithecia (Buchanan et al. 1981, Norconk 2007) indican además de Fabaceae a la familia Annonaceae como las de mayor importancia para estos primates; en nuestro caso Annonaceae se encuentra entre las siete principales familias con mayor número de especies de plantas alimenticias para $P$. aequatorialis.

Los resultados también indican que $P$. aequatorialis consumió frutos maduros e inmaduros, coincidiendo parcialmente con los obtenidos por Soini (1995), quien sostiene que $P$. isabela se alimentó únicamente de frutos maduros. Por otro lado, entre las semillas inmaduras consumidas por este primate, es oportuno destacar a Eschweilera spp. (Lecythidaceae) y Brosimun spp. (Moraceae), géneros que también son resaltados por Palminteri (2010) para $P$. irrorata, al sostener que esta especie a menudo se alimentaba de las semillas inmaduras de estas plantas.

Las plantas han adquirido una serie de adaptaciones en sus frutos para atraer a sus consumidores como el color, olor, sabor, tipo de pulpa, entre otras (Kricher 2008). En nuestro caso, el régimen alimenticio de $P$. aequatorialis estuvo mayormente compuesto por el mesocarpio maduro, coincidiendo así con los resultados de Buchanan et al. (1981) y Oliveira et al. (1985) para P. pithecia y Soini (1995) Aquino y Bodmer (2004) para $P$. isabela, pero contrasta con el de Palminteri (2010), quien sostiene que la dieta de $P$. irrorata estuvo conformada en más del $73 \%$ por semillas y solamente el $20 \%$ por la pulpa (mesocarpio). Por otra parte, el consumo de semillas, en particular de Eschweilera spp. y de otras fueron facilitados por los caninos que al igual que Cacajao spp. (Ayres 1989, Bartecki \& Heymann 1987, Cunha \& Barnett 1990, Aquino \& Encarnación 1999), Cebus y Chiropotes (Kinzey \& Norconk 1990) son fuertes y grandes para desgarrar el mesocarpio que usualmente se caracterizan por ser de textura dura. Es oportuno resaltar que al consumir semillas inmaduras estaría cumpliendo la función de controlador biológico de ciertas especies de plantas, entre ellas de Eschweilera spp, pero en el caso del consumo de frutos maduros con semillas pequeñas se convierten en importantes dispersores endozoocóricos de los géneros Pseudolmedia y Marcgravia y dispersores exozoocóricos de semillas medianas, entre ellas de $O$. bataua que después de consumir el mesocarpio son arrojadas algunos metros más allá del tallo.

La producción de frutos en el área de estudio fue durante todo el año, con un mínimo en la fructificación entre mayo-agosto, correspondiente a la época seca y un máximo entre noviembreabril, correspondiente al inicio y final de la época lluviosa; estos resultados son similares a los obtenidos por Aquino y Encarnación (1999) en el río Yavarí donde C. calvus ucayalii consumió la mayor diversidad de especies entre los meses de febrero a marzo y julio a octubre. Nuestros resultados también coinciden parcialmente con Palminteri (2010) para el Sur oriente donde P. irrorata consumió el mayor número de géneros de plantas entre febrero y mayo.

En el área de estudio, $P$. aequatorialis compite por el recurso alimenticio no solamente con mamíferos y aves de hábito diurno sino también nocturnos como Potos flavus y quirópteros frugívoros. Durante el seguimiento a los grupos de $P$. aequatorialis hemos observado árboles con abundantes frutos del cual se alimentaba este primate, pero al siguiente día los encontrábamos casi sin frutos, claro indicio de que otros animales también se alimentaban de estos recursos durante la noche, entre los que podrían considerarse a Aotus vociferans que según Puertas et. al., (1992) los frutos de Protium sp., forman parte de la dieta de este primate, pero que en este estudio no fue constatado por cuanto habita principalmente los bosques de terraza baja y de galería (Aquino \& Encarnación 1994). Entre las especies compartidas, los frutos de $P$. laevigata fueron los más requeridos por los competidores mamíferos y por algunas aves, entre ellas Ara spp., principales competidores de $P$. irrorata en el Sur oriente peruano (Palminteri 2010). Otra de las plantas alimenticias compartida con las aves fue $S$. morototoni, coincidiendo así con Parrini et. al. (2013), quienes indican que los frutos de esta planta sirven de alimento para muchas aves, entre ellas componentes de las familias Cracidae, Psittacidae, Ramphastidae e Icteridae.

\section{Agradecimientos}

El estudio fue financiado por Amazon Center por the Environmental Education and Research (ACEER) representado en Iquitos por la Licenciada Aura Murrieta, a quien expresamos nuestro agradecimiento. También agradecemos a Ricardo Zárate por su apoyo en la identificación de plantas. Nuestro reconocimiento a los Asistentes de campo Gilmer Montero Molano y Eli Díaz Rocha, quienes compartieron con nosotros momentos gratos y experiencias inolvidables. Finalmente, dejamos constancia de nuestra gratitud a la Blga. Emérita Tirado por su oportuna orientación durante la conducción del estudio.

\section{Literatura citada}

APG II. 2003. An update of the angiosperm phylogeny group classification for orders and families of flowering plants: APG II. Botanical Journal of the Linnean Society 141:399-436. http:// dx.doi.org/10.1046/j.1095-8339.2003.t01-1-00158.x

Aquino R., R.E. Bodmer. 2004. Plantas útiles en la alimentación de primates en la cuenca del río Samiria, Amazonia peruana. Neotropical Primates 12 (1): $1-5$.

Aquino, R. \& F. Encarnación. 1994. Owl monkey populations in Latin America: Field work and Conservation. In: J. S. Baer, R. E. Weller and I. Kakoma, eds. Aotus: The Owl Monkey. Academic Press, Inc. Pp. 59-95. http://dx.doi.org/10.1016/ B978-0-12-072405-5.50007-X

Aquino R. \& F. Encarnación. 1999. Observaciones preliminares sobre la dieta de Cacajao calvus ucayalii en el Nor-Oriente Peruano. Neotropical Primates 7(1): 1-5.

Aquino R., C. Terrones, R. Navarro \& W. Terrones. 2007. Evaluación del impacto de la caza en mamíferos de la cuenca del río Alto Itaya, Amazonía peruana. Revista Peruana de Biología 14(2): 181-186. http://dx.doi.org/10.15381/rpb.v14i2.1725

Aquino R., G. García \& E. Charpentier. 2013. Observaciones preliminares sobre aspectos ecológicos de Pithecia aequatorialis (Primates: Pitheciidae) en bosques del río Alto Itaya. En: R. Aquino, E. Lozano y D.E. Gil, eds. Mamíferos de la Cuenca del Río Alto Itaya, Amazonía Peruana. Saarbrücken (Germany), Editorial Académica Española. Pp. 226-250.

Ayres J.M. 1989. Comparative feeding ecology of the uakari and bearded saki, Cacajao and Chiropotes. Journal of Human Evolution 18: 21-24. http://dx.doi.org/10.1016/00472484(89)90101-2

Bartecki U. \& E.W. Heymann. 1987. Sightings of red uakaris, Cacajao calvus rubicundus, at the Rio Blanco, Peruvian Amazonia. Primate Conservation 8: 34-36.

Bowler M. \& R.E. Bodmer. 2011. Diet and food choice in Peruvian red uakaris (Cacajao calvus ucayalii): selective or opportunistic seed predation?. International Journal of Primatology 32: 1109-1122. http://dx.doi.org/10.1007/s10764-011-9527-6 
Buchanan D.B., R.A. Mittermeier \& M.G.M. Van Roosmalen. 1981. The Saki Monkeys, Genus Pithecia. In: A.F. Coimbra-filho and R.A Mittermeier, eds. Ecology and Behaviour of Neotropical Primates. Vol. 1. Academia Brasilera de Ciencias, Rio de Janeiro. Pp. 391-416.

Cunha A. Da \& A. Barnett, A. 1990. Sightings of the golden-backed uakari, Cacajao melanocephalus ouakary, on the upper Rio Negro, Brazil. Primate Conservation 11: 8-11.

Estrada A. 2006. Dispersión de semillas: interacciones entre plantas y primates, escarabajos coprófagos y otros mamíferos en la selva y su impacto en la dinámica y regeneración de la selva. Laboratorio de Primatología. http://www.primatesmx.com/ frugdisp.html. (Acceso 15/11/2014)

Garber P.A. 1993. Feeding ecology and behavior of the genus Saguinus. In: A.B. Rylands, ed. Marmosets and Tamarinds: systematic, behavior and ecology. Oxford University Press, Oxford. Pp. 273-295.

Heymann, E.W. \& U. Bartecki. 1990. A young saki monkey, Pithecia hirsuta, feeding on ants, Cachalots stratus. Folia Primatologica 55: 181-184. http://dx.doi.org/10.1159/000156514

Izawa K. 1975. Foods and feeding behavior of monkeys in the upper Amazon basin. Primates 16(3): 295-316. http://dx.doi. org/10.1007/BF02381557

Kinsey W.G. 1976. Positional behavior and ecology in Callicebus torquatus. Yearbook of Physical Anthropology 20: 468-480.

Kinzey W.G. 1977. Diet and feeding behavior of Callicebus torquatus. In: T.H. Clutton-Brock, ed. Primate ecology: studies of feeding and ranging behavior in lemurs, monkeys and apes. London, Academic Press. Pp. 127-151.

Kinzey W.G. \& A.H. Gentry. 1978. Habitat utilization in two species of Callicebus. In: R.W. Sussman, ed. Primate ecology: problem-oriented field studies. New York, Wiley. Pp. 89-100.

Kinzey W.G. \& M.A. Norconk. 1990. Hardness as a basis of fruit choice in two sympatric primates. American Journal of Physical Anthropology 81:5-15. http://dx.doi.org/10.1002/ ajpa.1330810103

Knogge C., E.R. Tirado \& E.W. Heymann. 2003. Effects of Passage through tamarin guts on the germination potential of dispersed seeds. International Journal of Primatology 24(5): 1121-1128. http://dx.doi.org/10.1023/A:1026236514523
Kricher J. 2008. Un compañero neotropical. Una introducción a los animales, plantas y ecosistemas del trópico del nuevo mundo. Segunda Edición, Modificada y Expandida. American Birding Association, Inc. Princeton University Press., Colorado. 462 pp.

Norconk M. A. 2007. Sakis, uakaris and titi monkey: behavioral diversity in a radiation of seed predators In: C.J. Campbell, S. Bearder, A. Fuentes, K.C. Mackinnon and M. Panger, eds. Primates in Perspective. New York, Oxford University Press. Pp. 123-138.

Oliveira J.M.S., M.G. Lima, C. Bonvincino, J.M. Ayres \& J.G. Feagle. 1985. Preliminary notes on the ecology and behavior of the guianan saki (Pithecia pithecia, Linnaeus 1766; cebidae, primate). Acta Amazonica 15 (1-2): 249-263.

Palminteri S. 2010. Determinants of primate distribution and abundance in South-western Amazonia, with a focus on baldfaced saki monkeys (Pithecia irrorata). Doctor of Philosophy Dissertation. School of Environmental Sciences, University of East Anglia, Norwich,UK. 203 pp.

Palminteri S., G.V. Powell \& C.A. Peres. 2012. Advantages of granivory in seasonal environments: feeding ecology of an arboreal seed predator in Amazonian forests. Oikos 121:1896-1904. http://dx.doi.org/10.1111/j.1600-0706.2012.20456.x

Parrini R., M.A. Raposo, J. Del Hoyo \& A. Ribeiro da Silva. 2013. Schefflera morototoni (Araliaceae) como importante recurso alimentar para as aves durante a estação seca na Amazônia central. Cotinga 35: 1-4

Peres C. A. 1993. Notes on the ecology of Buffy Saki Monkey (Pithecia albicans, Gray 1860): A canopy Seed-Predator. American Journal of Primatology 31: 129-140. http://dx.doi. org/10.1002/ajp.1350310205

Puertas P., R. Aquino \& F. Encarnación. 1992. Uso de alimentos y competición entre el mono nocturno Aotus vociferans y otros mamíferos, Loreto, Perú. Folia Amazónica 4(2): 147-156.

Riveros M. \& C. Ferreira. 2001. Comportamiento alimentario de una tropa de Pithecia pithecia (Mono viudo cara blanca), en una isla del embalse de Guri, Estado Bolívar. Sociedad Venezolana de Ecología. Ecotropicos 14(2): 57-64

Soini P. 1995. La dieta del mono huapo (Pithecia monachus). En: P. Soini, A. Tovar y U. Valdez, eds. Reporte Informe de Pacaya: Investigaciones en la estación Biológica Cahuana, 19791994. Pro Naturaleza - The Nature Conservancy, Lima, Perú. Pp. 273-278. 